\section{Anterior cutaneous nerve block for analgesia in anterior chest trauma: is the parasternal approach necessary?}

\author{
Santi Di Pietro', Benedetta Mascia², Giuliano Lo Bianco ${ }^{3}$, Stefano Perlini', \\ Giorgio Antonio lotti ${ }^{2}$
}

\author{
'Emergency Medicine Fellowship Program, Fondazione IRCCS Policlinico San Matteo, University of Pavia, \\ Pavia, Italy \\ ${ }^{2}$ Servizio Anestesia e Rianimazione 2, Fondazione IRCCS Policlinico San Matteo, University of Pavia, Pavia, \\ Italy \\ ${ }^{3}$ IRCCS CROB Centro di Riferimento Oncologico Basilicata, Rionero in Vulture, Potenza, Italy \\ ${ }^{4}$ Emergency Medicine Fellowship Program, Clinica Medica 2, Department of Internal Medicine, Fondazione \\ IRCCS Policinico San Matteo, University of Pavia, Pavia, Italy
}

In recent years, several techniques of regional anesthesia have been proposed to provide analgesia to the anterior thoracic cage; notably, most of these techniques require a parasternal approach. However, in this context, the potential role of a more common and well-established technique, namely the modified pectoral nerve block (known as PECS II block), has been poorly investigated. Here, we describe a case involving a patient with bilateral anterolateral multiple rib fractures associated with sternum fracture, who was successfully treated using bilateral PECS II blocks. Our experience indicates that the PECS II block can provide excellent analgesia in cases involving anterior rib and sternum fractures. Because it is easier to perform and may be safer than other parasternal techniques, the PECS II block should be considered when providing analgesia for traumatic injuries of the anterior thorax.

Keywords Rib fractures; Sternum; Nerve block; Anesthesia and analgesia

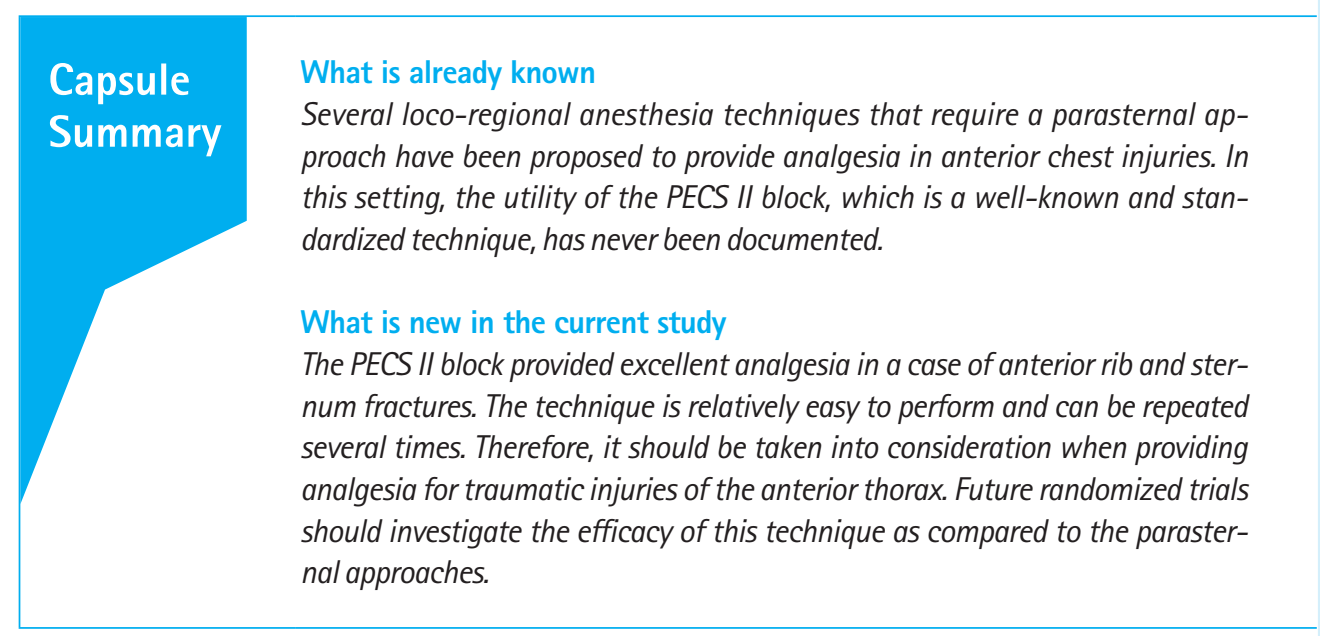

eISSN: $2383-4625$

Received: 19 December 2018

Revised: 28 January 2019

Accepted: 8 February 2019

Correspondence to: Santi Di Pietro Emergency Medicine Fellowship Program, Fondazione IRCCS Policlinico San Matteo, University of Pavia, Marchesi 24, Pavia 27100, Italy E-mail: santi.dipietro01@ universitadipavia.it ORCID http://orcid.org/0000-0001-6486-1924
This is an Open Access article distributed under the terms of the Creative Commons Attribution Non-Commercial License (http:// creativecommons.org/licenses/by-nc/4.0/). 


\section{INTRODUCTION}

In recent years, there has been increasing interest in the use of loco-regional anesthesia techniques to provide analgesia in patients with traumatic chest injuries. In particular, several techniques have been proposed to provide analgesia to the anterior thoracic cage, including transversus thoracic muscle plane block (TTP), parasternal intercostals nerve block (PSI), and subpectoral interfascial plane block (SIP). ${ }^{1-3}$ Although it is used in the routine practice of anesthesiologists with a number of surgical indications, the utility of the modified pectoral nerve block (PECS II) has been poorly investigated in the context of thoracic trauma. Here, we describe a case of a patient with bilateral anterolateral multiple rib fractures associated with sternum fracture, who was successfully treated using bilateral PECS II blocks. The local institutional review board authorized the submission of this manuscript (no. 20180046449). Written informed consent was obtained from all participants.

\section{CASE REPORT}

The local ambulance service was contacted for an 83-year-old man with a history of chronic obstructive pulmonary disease, who complained of dyspnea and cough that had persisted for a few hours. The patient was found in respiratory arrest; therefore, cardiopulmonary resuscitation was initiated by paramedics upon arrival. Cardiopulmonary resuscitation was interrupted after 10 minutes because of the return of spontaneous breathing. During resuscitation, the automated external defibrillator did not detect any shockable rhythm. Upon admission to the emergency department, chest computed tomography with an injection of contrast ruled out pulmonary embolism but showed bilateral pneumonia. In addition, it revealed the presence of multiple bilateral anterolateral rib fractures (II-VII on the right side, II-VI on the left side) associated with sternal body fracture; these were clearly a consequence of chest compression (Fig. 1). Lab findings showed troponin I level above the normal range $(0.3 \mathrm{ng} / \mathrm{mL})$.

A few days after admission to the Internal Medicine ward, the patient experienced chest pain that was poorly responsive to nonsteroidal anti-inflammatory drugs and paracetamol. Thus, the Internal Medicine staff sought a pain service consultation. Upon pain evaluation, the patient reported severe pain over the anterior thorax (numerical rating scale 7-8), which prevented him from coughing, moving, and breathing fully. We thus performed a bilateral PECS II blocks on each hemithorax, using $30 \mathrm{~mL}$ of $0.25 \%$ levobupivacaine combined with $6 \mathrm{mg}$ of dexamethasone $(15 \mathrm{~mL}$ injected between pectoralis major and minor muscles, $15 \mathrm{~mL}$ be-

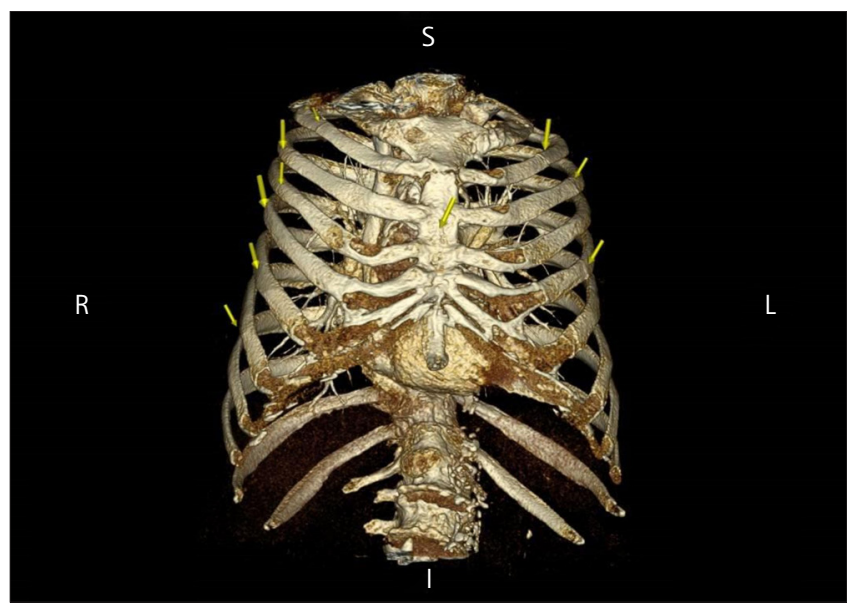

Fig. 1. Three-dimensional reconstruction of the patient's thoracic cage, showing sternal and rib fractures (some fractures are indicated by arrows). $S$, superior; $R$, right; I, inferior; $L$, left.

tween pectoralis minor and serratus muscle).

At 1 hour after the procedure, the patient reported only minimal discomfort above the sternum and fractured ribs (numerical rating scale 1-2). In addition, his capacity to cough was restored, and he could move and breathe deeply. Due to the recurrence of pain, the PECS II block was repeated, 48, and 80 hours after the first block. The patient's chest pain could be managed with nonsteroidal anti-inflammatory drugs and paracetamol. On day 20 after admission, his clinical condition improved, and he was transferred to another institution for rehabilitation.

\section{DISCUSSION}

In order to provide adequate analgesia to the anterior thoracic cage, as required in our patient, the target nerve structures for fascial plane blocks are the anterior cutaneous branches of the intercostal nerves. Successful block of these nerves can be achieved through TP block, which involves some technical difficulties and potential risks: first, the transverse thoracic muscle is thin, such that it is not always detectable by ultrasound; second, the target plane for this block lies deep in the intercostal space, near the pleura and internal mammary artery. ${ }^{1}$

Alternative strategies to the TP block include the SIP and PSI blocks: these techniques are similar in that they share the same plane for local anesthetic injection, which is situated between the pectoralis major muscle and external intercostal muscle. Additionally, the point of needle insertion is similar, lying $2 \mathrm{~cm}$ lateral to the sternal edge in the SIP, and immediately next to the sternum in the PSI. ${ }^{4,5}$ Therefore, it has been questioned whether these techniques should be regarded as two separate entities, or as vari- 

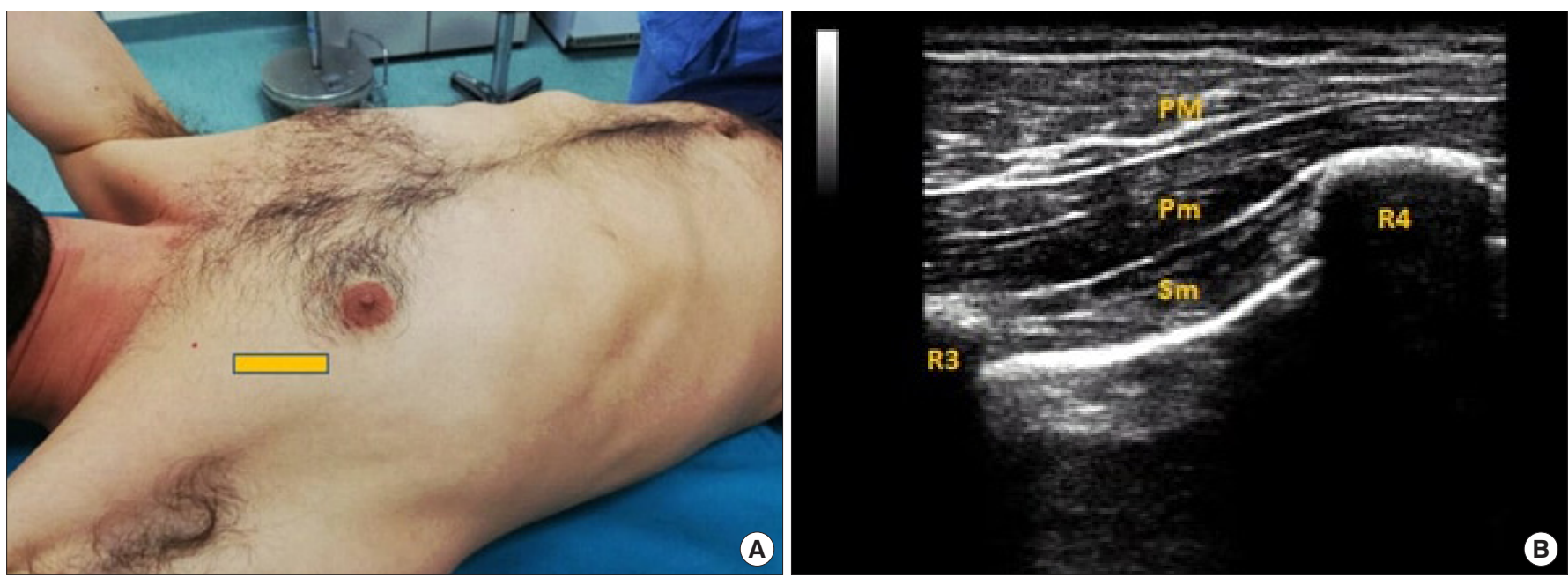

Fig. 2. Images show the correct positioning of the linear probe, which should be placed on the chest immediately below the lateral one-third of the clavicle (A). At this location, the anatomical landmarks can be easily identified: the pectoralis major (PM), pectoralis minor (Pm), and serratus anterior muscle $(\mathrm{Sm})$, as well as the third and fourth ribs $(\mathrm{R3}, \mathrm{R} 4)(\mathrm{B})$. The needle should be inserted using the in-plane approach, and the anesthetic should be injected both in the fascial plane between PM/Pm (PECS I) and in the fascial plane between Pm/Sm (PECS II). The procedure should be performed under sterile conditions. Pictures were taken from a healthy volunteer who provided written consent for publication.

ants of the same procedure. ${ }^{4,6}$ Other authors have previously highlighted the confusion resulting from this new nomenclature, thus suggesting that the techniques mentioned above are grouped under the common definition of 'parasternal intercostal plane blocks.'

The PECS II block was developed by Blanco et al. ${ }^{7}$ as a modification of the previously described PECS I, which consists of an injection of local anesthetic between the pectoralis major and minor muscles, in order to reach the lateral pectoral and medial pectoral nerves. In the modified technique (PECS II), an additional dose of anesthetic is injected between the pectoralis minor muscle and the serratus muscle, or immediately above the underlying rib.? (Fig. 2). This second injection aims to block important nerve structures: the intercostobrachial nerve, long thoracic nerve, and intercostal nerves III-IV-V-VI with their anterior branches. The PECS II technique was developed to provide complete analgesia during breast surgery and is now routinely employed by anesthesiologists for mastectomies, as well as placement of breast expanders and pacemakers. Although the site of injection in the PECS II block is more lateral on the chest wall, relative to that of the aforementioned parasternal techniques, the local anesthetic spreads along the fascial plane in all directions, thus reaching the anterior cutaneous branches more medially. This principle of local anesthetic diffusion applies to all interfascial plane blocks; however, the local spreading may be particularly enhanced in some interfascial planes than others, as recently noted by Elsharkawy et al. ${ }^{8}$ In fact, the deep fasciae of the pectoral region are thinner and more mobile than the fasciae of other anatomical sites; this may explain the ease with which local anesthetic dif- fuses along the thoracic wall, although this hypothesis must be confirmed in future research. ${ }^{8}$ In the clinical case presented here, bilateral PECS II blocks provided excellent pain control for multiple anterolateral rib and sternum fractures, suggesting that satisfactory block of the anterior branches of intercostal nerves does not necessarily require a parasternal approach.

This technique offers several advantages relative to the use of other parasternal intercostal plane blocks (TTP, SIP, and PSI): First, in PECS II block, the needle does not enter the intercostal space, as it remains above the ribs. Second, handling of the probe and needle-probe alignment are much easier in the lateral chest immediately above the pectoral muscles, compared to the sternal proximity. Moreover, PECS II block is a technique already within the armamentarium of most anesthesiologists; therefore, it is likely easier to teach non-anesthesiologist physicians who wish to approach the field of regional anesthesia (e.g., emergency physicians). Therefore, in the context of anterior chest trauma, PECS II block is likely to be technically easier and potentially safer than other parasternal approaches.

PECS II block exhibited excellent analgesia for a patient with anterior rib and sternum fractures. The technique is relatively easy to perform and can be repeated several times with a minimal risk of local anesthetic toxicity, as large volumes are used with low concentrations of anesthetic. Therefore, this approach should be considered when providing analgesia for traumatic injuries of the anterior thorax. Future randomized trials should investigate the efficacy of this technique, compared to that of other parasternal approaches. 


\section{CONFLICT OF INTEREST}

No potential conflict of interest relevant to this article was reported.

\section{ACKNOWLEDGMENTS}

The authors would like to thank Dr. Francesca Rella and Dr. Luigi Sammarchi for their help with image editing.

\section{REFERENCES}

1. Ueshima $H$, Kitamura A. Blocking of multiple anterior branches of intercostal nerves (Th2-6) using a transversus thoracic muscle plane block. Reg Anesth Pain Med 2015;40:388.

2. Ohgoshi Y, Ino K, Matsukawa M. Ultrasound-guided parasternal intercostal nerve block. J Anesth 2016;30:916.
3. Raza I, Narayanan M, Venkataraju A, Ciocarlan A. Bilateral subpectoral interfascial plane catheters for analgesia for sternal fractures: a case report. Reg Anesth Pain Med 2016;41: 607-9.

4. Ueshima H, Otake H. Similarities between parasternal intercostal nerve block and subpectoral interfascial plane block. J Anesth 2017;31:476.

5. Ohgoshi Y, Ino K, Matsukawa M. In reply: Similarities between parasternal intercostal nerve block and subpectoral interfascial plane block. J Anesth 2017;31:477.

6. Chin KJ. An anatomical basis for naming plane blocks of the anteromedial chest wall. Reg Anesth Pain Med 2017;42:414-5.

7. Blanco R, Fajardo M, Parras Maldonado T. Ultrasound description of Pecs II (modified Pecs I): a novel approach to breast surgery. Rev Esp Anestesiol Reanim 2012;59:470-5.

8. Elsharkawy H, Pawa A, Mariano ER. Interfascial plane blocks: back to basics. Reg Anesth Pain Med 2018;43:341-6. 